\title{
SUPERBEND PROJECT AT THE ADVANCED LIGHT SOURCE*
}

\author{
D. Robin, A. Biocca, B. Brown, W. Byrne, D. Calais, M. Chin, D. Colomb, C. Corradi, J. De Vries, \\ M. Fahmie, A. Geyer, J. Harkins, S. Jacobson, J. Krupnick, S. Marks, J. McDonald, R. Mueller, \\ F. Ottens, A. Paterson, P. Pipersky, A. Ritchie, R. Schlueter, J. Spring, C. Steier, C. Taylor, C. Timossi, \\ W. Thur, A. Wandesforde, J. Zbasnik, LBNL, Berkeley, 94720, USA, \\ J. Chen, B. Wang, WANG NMR, Livermore CA, 94550
}

\begin{abstract}
To satisfy the demand for more high energy, high brightness X-ray sources at the Advanced Light Source (ALS), a plan is in place to replace three 1.3 Tesla normal conducting bending magnets with three 5 Tesla superconducting magnets (Superbends) in the year 2001. This will result in 12 new x-ray beam lines (four from each superbend) for users. The Superbend sources will be an order of magnitude higher in x-ray brightness and flux at $12 \mathrm{keV}$ than the conventional 1.3 Tesla bending magnets. The Superbend project is a major upgrade to the ALS where the 3 superconducting magnets will be an integral part of the machine lattice. In this paper we discuss the current status of the Superbend projectas well as precomissioning studies prior to the 2001 installation for users.
\end{abstract}

\section{INTRODUCTION}

There has been a rapidly growing demand for more bright x-ray sources with photon energies of 10 to $30 \mathrm{keV}$. At the ALS this demand has come from several areas both from existing ALS user communities, such as the protein crystallography community, as well as potential new user communities, such as the x-ray tomography and powder diffraction communities. In particular the protein crystallography community is growing so rapidly that their needs far exceeds the capacity of the present beamlines.

To provide new hard $\mathrm{x}$-ray sources, the ALS is planning to replace three of the thirty six, 1.3 Tesla, normal conducting, 10 degree, bending magnets with three, 5 Tesla, superconducting, 10 degree, bending magnets (Superbends). The three Superbends will provide twelve new beam lines for users (four from each bend).

Specifically the Superbends will be placed in three of the twelve ALS sectors $(4,8$, and 12). The arc lattice consists of a triple bend achromat. Fig. 1 shows how each of the 3 sectors will be modified to include Superbends. A typical sector without Superbends can be seen in Fig. 1 (top) and one modified to include Superbends is shown in Fig. 1 (bottom). One sees that the central dipole, B2, in the sector is replaced by a Superbend. Unlike the normal dipoles, the Superbends do not have a large quadrupole focusing component. Two new quadrupoles QDA1 and QDA2 are added to the lattice and the QFA quadrupoles in a Superbend sec-

* This work was supported by the Director, Office of Energy Research, Office of Basic Energy Sciences, Materials Sciences Division of the U.S. Department of Energy, under Contract No. DE-AC03-76SF00098. tor are put on separate power supplies. It is necessary to make this change in the quadrupole configuration in order to better match the Superbend sectors to the non Superbend sectors.

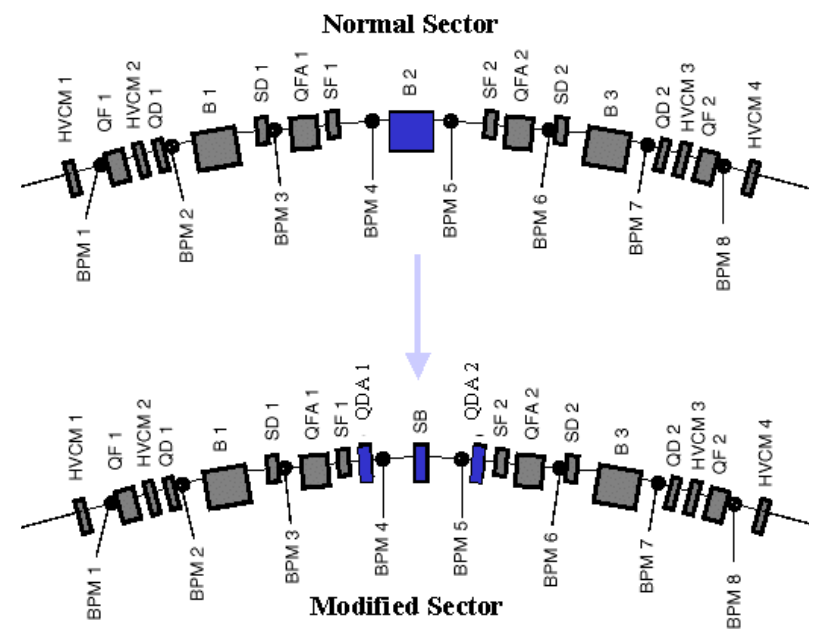

Figure 1: Magnetic layout of a normal (top) and modified (bottom) sector

The center bend of a triple bend achromat is the best choice for a high brightness bend magnet source because the beta functions are very small in both the horizontal and vertical planes [1].

At an electron beam energy of $1.9 \mathrm{GeV}$, the Superbends will have a a critical photon energy of $12 \mathrm{keV}$ - as opposed to a critical photon energy of $3.1 \mathrm{keV}$ for the 1.3 Tesla normal conducting bends. The increase in critical energy means that in in both flux and brightness the Superbend is almost an order of magnitude larger than the normal bend magnet at $10 \mathrm{KeV}$ and almost two orders of magnitude larger at $20 \mathrm{KeV}$. A comparison of the brightness of the Superbends versus the normal bends can be seen in Fig. 2.

\section{SUPERBEND PROJECT}

The idea of placing superconducting magnets in the ALS was conceived by Alan Jackson (LBNL) and Werner Joho (SLS) in the early 1990s. A study was carried out by LBNL's Superconducting Magnet Group to design and produce a coil and core of a superconducting magnet that would produce the desired peak and integral fields while accomodating the ALS vacuum chamber. In 1997 a prototype of the magnet core and coils was built by Wang NMR 


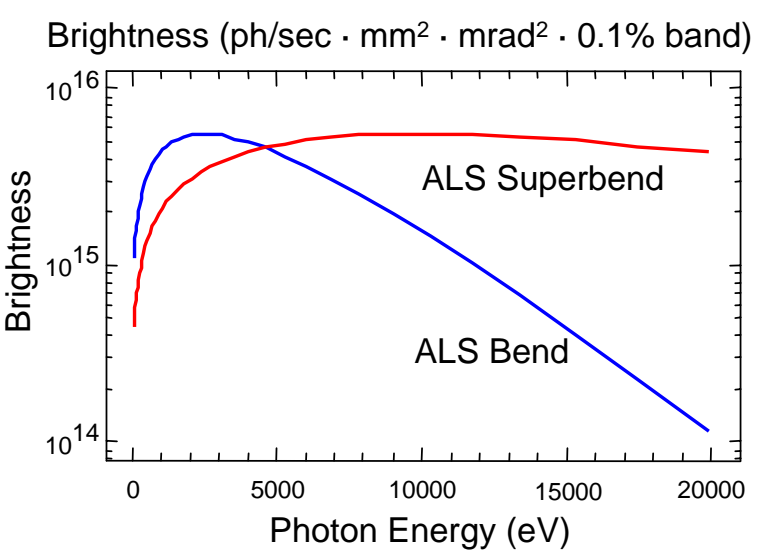

Figure 2: Comparison of brightness of the normal and Superbends. The brightness is calculated assuming a vertical emittance of $0.1 \mathrm{~nm}$-rad and a horizontal emittance of 6 $\mathrm{nm}$-rad for the normal bends and 12nm-rad for the Superbends.

in conjuction with LBNL and reached design current without training quenches[2]. In 1998 a test cryostat was built and static and dynamic magnetic fields were measured[3]. These results together with the fast growing user community prompted the ALS to form a Superbend project team.

\subsection{Beam Dynamics Studies}

In 1998 tracking simulations had been performed to assess the impact of the Superbends on the beam dynamics. The initial studies were done assuming an isomagnetic model for the Superbends. The results showed that for our nominal tune there was no major impact on injection and beam lifetime at $1.9 \mathrm{GeV}[1]$. However there was concern that the isomagnetic model was inadequate for tracking. This is because the Superbend field is far from isomagnetic.

In 1999 a full 3-D field map of the magnet was included in the storage ring model. Tracking studies were repeated and the results were not significantly different from the results with the isomagnetic Superbend model. Also accelerator simulations were done to study the effect of ramping and electron beam stability.

\subsection{Cryosystem}

In 1999 a conceptual design for the Superbend cryosystem was made based upon two key features: a Gifford McMahon cryocooler and high temperature superconducting (HTS) leads. The Gifford McMahon cryocooler was chosen because it is regarded as more reliable than cryocoolers with Joule-Thomson valves. HTS leads have a very low heat leak compared with normal conducting leads which is essential for operation with a low power cryocooler.

In FY99 a test cryosystem was built to test the performance of the cryocooler and the HTS leads. A 1.5 W Sum- itomo cryocooler and a set of American Super Conductor HTS leads were purchased. These were incorporated into the previously built cryosystem[4].

In all aspects the system performed well. The cryocooler was very easy to set up and operate and it had sufficient capacity for cooling the Superbend. Measured heat leaks from the leads were low and in agreement with the estimated heat leaks. The ASC leads (2223 with Ag alloy matrix) were found to be very stable and robust against temperature excursions. The results of these tests ensured us that the design concept for the cryosystem is sound and the system should perform reliably.

\subsection{Preparation for Superbend Installation}

In 1999 the complete Superbend design was finished and Wang NMR was commissioned to construct 4 Superbend articles ( 3 plus a spare)[5]. Concurrently the project team outlined its strategy to transition to Superbend operation. It is clearly desirable to minimize the amount of unscheduled operational downtime and poor operation. Therefore the project team has adopted the strategy of precommissioning as many subsystems (with and without beam) as possible prior to the actual installation of the Superbends. As a result the installation for the Superbend project is split into two medium length (few week) shutdowns rather than one long shutdown. In the first shutdown (which occurred in March 2000) all major components of the project, excluding the actual Superbend magnets were installed. That included machining the vacuum chambers, installing the 6 new quadrupoles (QDAs), pulling cables for the Superbend magnets, recabling the existing quadrupoles (QFAs), installing new power supplies, and moving 11 magnet power supplies over to a new control system. This was the single largest modification of the control system since the ring was first commissioned. In spite of all these modifications, the shutdown and ring startup went well with beam being stored and ramped within several hours after startup.

There have been ongoing studies to ensure that the installation of the Superbends does not impact the performance of the storage ring. In particular a concern was that the Superbends will perturb the natural 12 fold symmetry of the storage ring which could excite resonances in the beam motion with a subsequent reduction in the injection efficiency and lifetime. Since the installation of the QDA quadrupoles it has been possible to experimentally perturb the periodicity of the storage ring and simulate (to some degree) the impact of the Superbends on operation [6]. In particular, dynamic momentum acceptance measurements were made. The results show that even with the symmetry breaking of the Superbends the ALS can be operated with negligible performance degradation.

Concurrently in 2000, significant progress was made in the construction of the 4 Superbend magnet/cryostat assemblies. The first of four Superbend magnets was successfully built by Wang NMR [7]. A picture of the first magnet cryostat system can be seen in Fig 3 . 

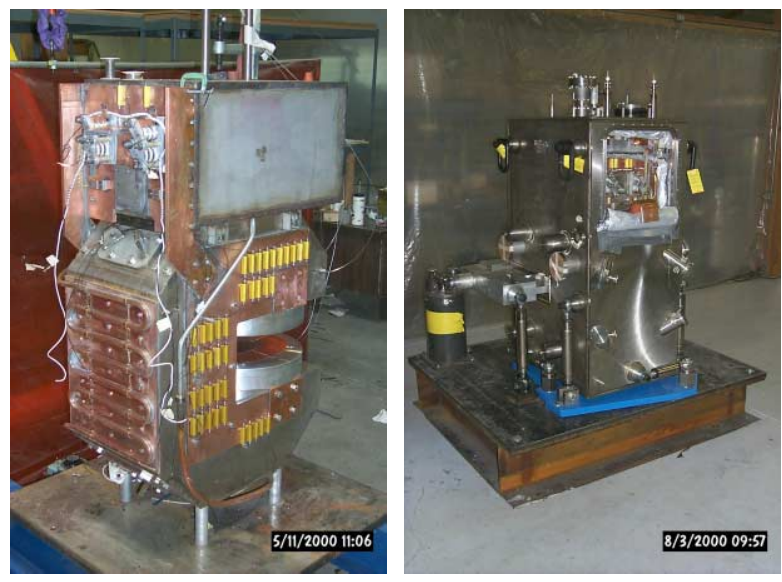

Figure 3: First magnet/cryostat assembly.

First Article On August 24, the first Superbend magnet was successfully charged and ramped to full current without training. In subsequent tests the magnet has been ramped between injection and operation energy in under 2 minutes. This is similar to the ramping time that we presently operate with.

Other performance tests of the first Superbend assembly have gone well. Preliminary measurements of the magnetic field reveal that the predicted transfer function between magnet currents and integrated dipole field agreed. Extensive vibration measurements have been carried out indicating that there will be no significant increase in beam motion due to Superbend vibrations. Initial thermal tests of the first Superbend reveal larger than desirable heat leaks. Modifications were made that have improved thermal performance of the magnet. After modifications, the thermal performance met requirements. In addition to the cryogenic performance tests of the first Superbend there have been extensive magnet measurements (multipoles and transfer functions), fiducialization of the first magnet, and power supply tests. In Fig. 4 a longitudinal Hall probe scan is displayed. The measurement was made at an excitation current of $300 \mathrm{Amps}$ which corresponds to $1.9 \mathrm{GeV}$ operation.

\subsection{Remaining Work}

The final 3 magnet cryostat assemblies have been completed and are currently undergoing characterization studies (similar to the first Superbend). After characterizing the remaining Superbends, they will be installed in the storage ring during a 6 week installation and commissioning period in the fall of 2001.

\section{ACKNOWLEDGEMENTS}

The authors would like to thank Greg Portmann, Ying Wu, and Kurtis Nishimura, T. Henderson, J. Hinkson, and E. Hoyer for their valuable contributions to the project.

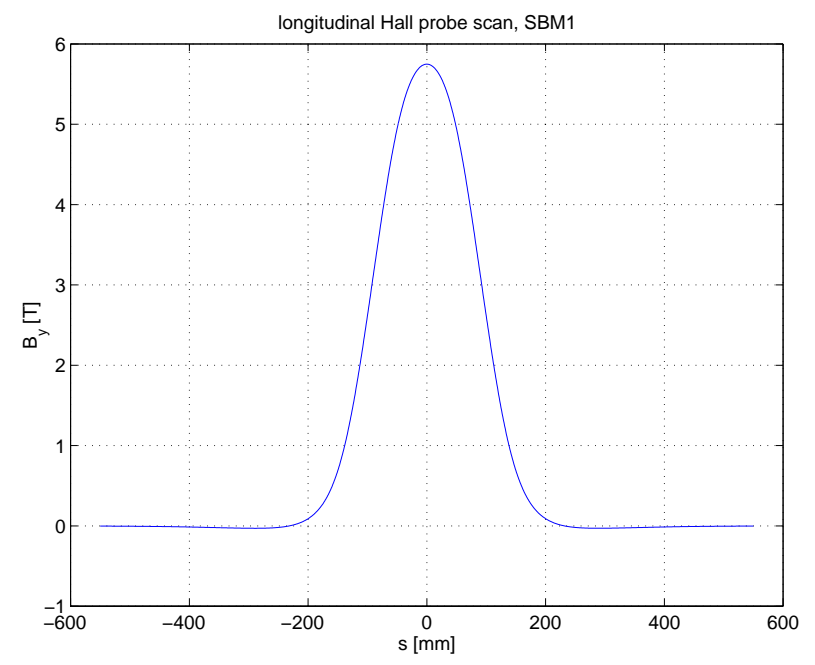

Figure 4: Magnetic field versus longitudinal position at 300 Amps.

\section{REFERENCES}

[1] H. Nishimura and D. Robin, "Impact of Superbends at the ALS", proceedings of the 1999 Particle Accelerator Conference, (1999)

[2] C. E. Taylor and S. Caspi, "A 6.3 T Bend Magnet for the Advanced Light Source”,IEEE Trans. Magnetics 32, No. 4, 1996, pp. 2175-2178.

[3] C. E. Taylor, et al., "Test of a High-Field Magnet for the ALS", Transactions on Applied Superconductivity 9, No. 2, 1999, pp. 479-482.

[4] J. Zbasnik, et. al. "Test of a GM cryocooler and high Tc leads for use on the ALS Superbend magnets", published in the proceedings of the 1999 CEC, Montreal, Quebec, Canada, July 1999.

[5] "Superbend Magnet System conceptual Design Report", Lawrence Berkeley national Laboratory Publication, PUB5457, Apr. 2000

[6] C. Steier, et al., "Understanding the Dynamic Momentum Aperture of the Advanced Light Source", Proceedings of the 2001 Particle Accelerator Conference, Chicago, IL (2001)

[7] J. Zbasnik, et al., "ALS Superbend Magnet System", Proceedings of the 2000 Applied Superconductivity Conference, Norfolk, VA, September 2000. 